\title{
Impfpflicht im Zwielicht
}

\author{
Die Diskussion um eine mögliche COVID-19-Impfpflicht verwirrt. \\ Zwischen politischer Zurückhaltung und Radikalität, echten Ängsten und \\ irrealen Mythen sind drei Fakten unverrückbar: Eine Verpflichtung, sich impfen zu lassen, \\ ist rechtlich möglich, ohne Fremdschutz ist sie schwer zu argumentieren \\ und solange nicht genügend Impfstoff zur Verfügung steht, \\ bleibt alles Theorie. Alexandra Keller
}

Diese Ohnmacht kam höchst ungelegen. Kurz nachdem Tiffany Dover Mitte Dezember 2020 gegen COVID-19 geimpft worden war, wurde ihr schwindelig und sie verlor kurz das Bewusstsein. Bald konnte die ganze Welt Zeuge des unangenehmen Aussetzers der jungen Krankenschwester des CHI Memorial Krankenhauses in Chattanooga, einer Stadt im US-Bundesstaat Tennesee, werden. Die Ohnmacht verwandelte den als Werbung für die COVID-19-Impfung gedachten Medienauftritt ins Gegenteil. Denn Impfgegner nutzten das Video geschwind, um zu belegen, dass der Biontech/Pfizer-Impfstoff nicht sicher sei. Weltweit gras-

\section{Medizinrechtsexperte Karl Stöger:
„Beim Thema Impfpflicht zucken alle aus."}

sierte der Schwindelanfall im Netz, auch in Österreich, wo die FPÖ den kurzen Film flugs auf ihre Homepage stellte und mit ihrer Kampagne „Klares NEIN zum Impfzwang“ verknüpfte. Die heimischen Rechtspopulisten bringen eine potenzielle COVID19-Impfpflicht aus polittaktischen Gründen ins Zwielicht, jonglieren in Trump'scher Manier mit vermeintlichen Fakten und verirren sich dabei im rechtlichen Dschungel. Etwa wenn der freiheitliche Nationalratsabgeordnete Christian Ragger behauptet: „Impfpflicht ist ganz klar verfassungswidrig!“ Denn damit liegt er ganz klar falsch.

\section{Rechtliche Säulen}

Abseits aller Unsicherheiten und Unklarheiten, denen die Bundesregierung im Falle der COVID-19-Impfung nicht wirklich mit entsprechend ehrlicher Information und Aufklärung begegnet, stehen die rechtlichen Säulen, auf denen eine eventuelle Impfpflicht thront, recht felsenfest im österreichischen Staatsgebilde. „Die Verfassung steht einer verhältnismäßig ausgestalteten Impfpflicht jedenfalls nicht grundsätzlich entgegen: Denn der damit einhergehende Eingriff in das Recht auf Privatleben ge- mäß Art. 8 EMRK kann bei entsprechender Gefährdungsprognose durchaus durch den Schutz der Gesundheit im Sinne des Art. 8 Abs. 2 EMRK gerechtfertigt sein, ohne dass dafür erst der Eintritt einer großflächigen Epidemie abgewartet werden müsste. [...] Und was die neuerdings aufgetauchten Zweifel an der Regelungskompetenz für eine - wie auch immer ausgestaltete - Impfpflicht betrifft, ergibt sich die Zuständigkeit des Bundesgesetzgebers aus Art. 10 Abs. 1 Z 12 B-VG, der generelle Maßnahmen zur Bekämpfung von Infektionskrankheiten einschließt. Das gilt nicht nur für Impfpflichten der Bevölkerung, sondern auch des Gesundheitspersonals: Gegenüber Personen, die sich ,berufsmäßig“ mit der Krankenbehandlung oder Krankenpflege beschäftigen, können Schutzimpfungen gemäß $\$ 17$ Abs. 3 Epidemiegesetz sogar im Verordnungsweg angeordnet werden“, hielt Christian Kopetzki, Professor für Medizinrecht an der Universität Wien in Recht der Medizin, einem periodisch im Manz-Verlag erscheinenden juristischen Journal, fest. 2017 tat er das, als Experten bezüglich der bedrohlich angestiegenen Masernfälle in Österreich „Gefahr im Verzug“ orteten und eine Impfpflicht angedacht wurde.

\section{Nur Eigenschutz ist Autonomie}

„Daran hat sich nichts geändert", stellt Karl Stöger, ebenfalls Professor für Medizinrecht an der Universität Wien, klar, dass die Corona-Pandemie mit denselben juristischen Vorzeichen betrachtet werden muss. Die bedrohliche Lage hat die juristischen Säulen nicht verrückt. Die österreichische Bundesregierung kann eine COVID-19-Impfpflicht beschließen, wenn sie will.

Ein nicht unerhebliches Argument, das derzeit rein faktisch gegen eine Impfpflicht spricht, ist der Umstand, dass schlichtweg zu wenig Impfstoff zur Verfügung steht, um die Bevölkerung zur Impfung zu verpflichten. Laut WHO müssen bis zu 70 Prozent der Bevölkerung immun beziehungsweise geimpft sein, um eine virusvernichtende Herdenimmunität zu erreichen. Auch dabei spielt die nach wie vor unbeantwortete Gretchenfrage im $\mathrm{Zu}$ sammenhang mit dem Wirkungsspektrum der derzeit zugelassenen COVID-19-Impfstoffe eine entscheidende Rolle: Die Frage, ob 
Hepatitis B und Pneumokokken für alle medizinischen Personengruppen zu Hepatitis A und Meningokokken für ausgesuchte medizinische Berufsgruppen. Eine dezidierte Impfpflicht für das Personal des Gesundheitswesens gibt es nicht, doch wird versucht, diese mit der Formulierung „moralische Verpflichtung“ zu erreichen. „Das ist politisch ein bissl feig. Das Ministerium weiß, dass eine Impfpflicht nach $\$ 17$ Epidemiegesetz angeordnet werden könnte. Das wollen sie aber nicht, weil beim Thema Impfpflicht alle auszucken“, meint Medizinrechtler Stöger. Die „moralische Verpflichtung“ hält er für einen Versuch, darauf hinzuweisen, wie wichtig die Impfungen sind, nach dem Motto: Menschen, die Menschen helfen, heilen sie und gefährden sie nicht.

Die sanft formulierte Anleihe aus der Medizinethik gibt rechtlich nicht allzu viel her. Lassen sich Mitarbeiter in Gesundheitsund Pflegeeinrichtungen jedoch nicht entsprechend impfen, kann sich das auf das Dienstverhältnis auswirken oder darauf, ob es überhaupt zu einem Verhältnis kommt. „Die Verweigerung einer Impfung kann im Gesundheitswesen ein Grund dafür sein, dass ich den Job nicht bekomme“, sagt Stöger.

\section{Bei Weigerung Versetzung}

Wer auf einer Kinderstation arbeiten, sich aber beispielsweise nicht gegen Masern impfen lassen will, muss seinen Berufswunsch möglicherweise umformulieren. Anders ist es bei denen, die bereits angestellt sind. In der 2017 erschienenen Informationsbroschüre Impfungen des Gesundheitspersonals - Rechtliche Aspekte hielt das Gesundheitsministerium fest, dass Mitarbeitern, die Impfungen ablehnen oder ihren Impfstatus nicht offenlegen, „eine weniger gefahrengeneigte Tätigkeit zuzuweisen“ sei. Versetzung lautet demnach das erste Instrument, dessen sich die Arbeitgeber bedienen können. Kündigung lautet das ultimative. „Die Versetzbarkeit endet dort, wo überwiegend keine dem jeweiligen Berufsbild entsprechenden Tätigkeiten mehr wahrgenommen werden oder dies ein faktisches Berufsverbot darstellt. Die Weigerung, eine nicht oder weniger gefahrengeneigte Tätigkeit aufzunehmen oder den Impfstatus offenzulegen - im Übrigen auch die wahrheitswidrige Information darüber - berechtigt zur Entlassung“, heißt es in der Broschüre des Ministeriums. Eine Kündigung ist rechtlich heikel. Dass Mitarbeiter, die eine Impfung verweigern, eine möglicherweise nachteilige Versetzung in Kauf nehmen müssen, ist naheliegender.

Nur langsam wächst die Bereitschaft des Gesundheitspersonals, sich gegen COVID-19 impfen zu lassen. Zu groß ist auch bei ihnen die Angst vor möglichen Impfschäden. Zu verschwindend sind offenkundig auch in diesem Bereich die ernst gemeinten Versuche, aufzuklären, zu informieren und auf die Ängste einzugehen. „Fünf Sätze reichen da nicht. Man darf die Menschen

\section{Der Fremdschutz ist das Argument für die Impfpflicht."}

nicht für blöd verkaufen“, sagt Karl Stöger und ergänzt: „Irgendwo habe ich gelesen, wenn sich Andreas Gabalier nicht impfen lässt, haben wir ein Problem. Wenn Gabalier aber sagt, die Impfung ist super, dann rennt es. Das stimmt wahrscheinlich." Vergleichsweise schlecht scheint da die Wahl jener Krankenschwester gewesen zu sein, deren öffentlichkeitswirksame Ohnmacht in Folge der COVID-19-Impfung im Dezember 2020 die Netze heißlaufen ließ. Nach dem Schwindelanfall wurde bekannt, dass sie eine Vorgeschichte mit überempfindlichen vasovagalen Reaktionen hat und wegen Schmerzen immer mal wieder das Bewusstsein verliert. Wissenschaftler hielten in dem Zusammenhang fest, dass die Ohnmacht durch den Impfvorgang und nicht durch den Impfstoff ausgelöst wurde. Na dann.

\section{ALEXANDRA KELLER}

kelleragesundheitswirtschaft.at

\section{DIE PRIMÄRVERSORGUNGS- EINHEIT ALS FUNDAMENT DER VERSORGUNG!}

In unseren Projekten als Gesundheitsplaner - gerade in diesen herausfordernden Zeiten - vertreten wir die Meinung, die wirksame Primärversorgung zu stärken. Die Dringlichkeit für den raschen Ausbau von Primärversorgungseinheiten wird nicht nur in den aktuellen Gesundheitsplänen ersichtlich, sondern vor allem auch aus der Erfahrung der Bewältigung der Pandemie notwendig.

Für uns ist es daher wichtig, wirkungsvolle Gesamtlösungen nachhaltig zu entwickeln. Ob Planung, Umsetzung oder Evaluierung - wir stehen Ihnen zur Seite!
Wir bringen's auf den Punkt.

Kontaktieren Sie uns unter: wbayer@wbayer.at www.wbayer.at

MMag. Wolfgang Bayer Geschäftsführer Büro Linz:

Stelzhamerstraße 2/24 4020 Linz

M: +43 6644682074

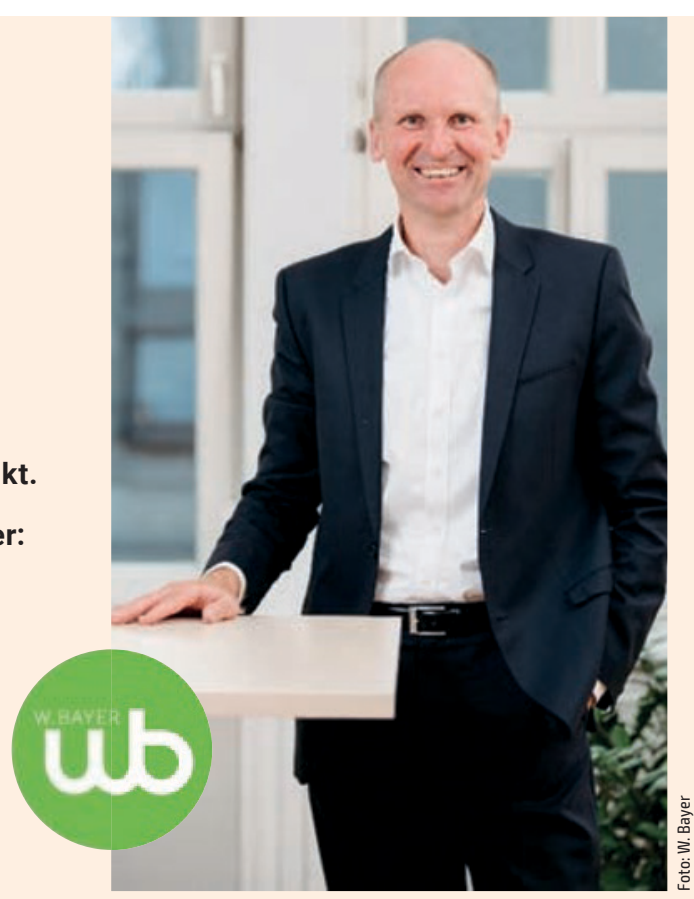

